\title{
Molecular marking in breeding Brassica oleracea $L$. for resistance to Xanthomonas campestris pv. campestris
}

\author{
Yuliya Makukha* and Elena Dubina \\ Federal Scientific Rice Centre, bldg. 3, pos. Belozerniy, Krasnodar, 350921, Russia
}

\begin{abstract}
In the presented study, we have studied polymorphism of 30 microsatellite loci in terms of resistance to black rot (Xanthomonas campestris). Three SSR markers (O110-C01, O111-H06, BoESSR 726) were identified, showing polymorphism in white cabbage forms with contrasting resistance to black rot. Also, part of the F2 breeding material was analyzed using markers Ol10-C01, Ol11-H06. It was found that they reveal all types of allelic state of genes in a segregating population in accordance with the Mendelian rule of segregation, which makes these markers suitable for further use in studies on the basis of cabbage resistance to black rot.
\end{abstract}

\section{Introduction}

Black rot of white cabbage is an extremely destructive disease that leads to significant yield losses (more than 50\%) worldwide in the presence of favorable conditions for the bacteria [1]. This disease is associated with damage to the plant vascular system, in which the bacterium Xanthomonas campestris pv. campestris (Pam.) Dowson penetrates the xylem, colonizes the mesophyll and then spreads through the tissues of the entire plant. Further, chlorous $\mathrm{V}$-shaped spots are formed, leading to necrosis of plant tissue and death of the entire plant $[2,3,4]$. Compiling an algorithm of measures to prevent this disease is an extremely laborious process, since the racial composition of the pathogen is very diverse. To date, 11 Xcc races, have been identified, of which in the south of Russia the most widespread and destructive are races 1 and 4 [5, 6, 7]. In addition to the diversity of pathogen races, the limiting factor in white cabbage $(B$. oleracea) breeding is the fact that sources of resistance to the main damaging Xcc races (1 и 4) are more often present in A and $\mathrm{B}$ genomes (B. rapa and B. nigra) and rarely are found in the $\mathrm{C}$ genome (B. oleracea) . For example, Sharma et.al. (2016) have already identified the gene for resistance to 1 race of the Xcalbc pathogen and there are effective markers for their identification [8]. For $B$. oleracea, the study of inheritance patterns of black rot resistance is difficult, since it was found that it is polygenic in nature, since resistance genes and QTLs were identified in different linkage groups [9]. Lee (2015) mapped a total of 14 QTLs associated with Xcc resistance on eight chromosomes out of nine in $B$. oleracea, four of them being the main

\footnotetext{
*Corresponding author: makyxa69@mail.ru
} 
loci affecting plant resistance [10]. Tonu et al. (2013) identified the most significant QTLs: major XccBo (Reiho) 1, minor XccBo (Reiho) 2 and $X c c B o(G C 1)$, as well as the markers closest to these loci [2]. Afrin et al. (2018) tested some of these markers (9 SSR and 1 InDel) on 27 inbred cabbage lines resistant to different races of the pathogen . The results of comparison of molecular screening and phytopathological tests made it possible to select five markers capable of distinguishing resistant forms from affected ones [11]. However, to date, it has not been possible to develop an integral DNA marker system for such a complex polygenic trait.

Thus, due to the lack of a universal marker system for the reliable identification of resistance genes to Xanthomonas campestris, molecular genetic research related to the search and development of effective molecular markers remains an urgent problem for the genetics and breeding of white cabbage, the solution of which will make it possible to reduce the breeding process, as well as to obtain valuable stable genotypes of white cabbage with the desired biological properties. The use of molecular marking has already made it possible to solve the problem of identifying donors of resistance to pathogens on other crops, in particular on rice [12, 13].

\section{Materials and methods}

PCR analysis of the studied white cabbage plants was carried out on the basis of the laboratory of information, digital and biotechnologies of FSBSI "Federal Scientific Rice Centre" in 2020. The material of the study were the contrasting forms of white cabbage: resistant (isogenic line 269-Yas12p-2) and susceptible (isogenic line Pi714) to black rot, selected in the department of vegetable and potato growing of FSBSI "Federal Scientific Rice Centre", as well as F2 plants of the hybrid combination 269-Yas x Pi714 obtained as a result of self-pollination of F1 plants. DNA from cabbage leaves was isolated according to the scheme of Murray and Thompson [14] using cetyltrimethylammonium bromide $(\mathrm{CTAB})$ as a lysis buffer for plant cells.

When carrying out molecular genetic studies, neutral codominant microsatellite (SSR) markers were used, taken from the database on the website www.VegMarks.ru, developed to assess polymorphism in white cabbage plants, as well as SSR markers from Afrin et al. (2018) [9].

Separation of amplification products was carried out using a $2 \%$ agarose gel with the addition of ethidium bromide at a voltage of $130 \mathrm{~V}$ for 1 hour, followed by their visualization in ultraviolet light.

\section{Results of study}

A total of 30 microsatellite markers localized in different chromosomes were tested during DNA analysis of plant forms contrasting in resistance to black rot, 3 of which (Ol10-C01, O111-H06, BoESSR 726) revealed polymorphism in the studied samples. Allelic diversity at the O110-C01 and O111-H06 loci was demonstrated in the previous work [15]. The polymorphism at the BoESSR 726 locus can be seen from Figure 1. 


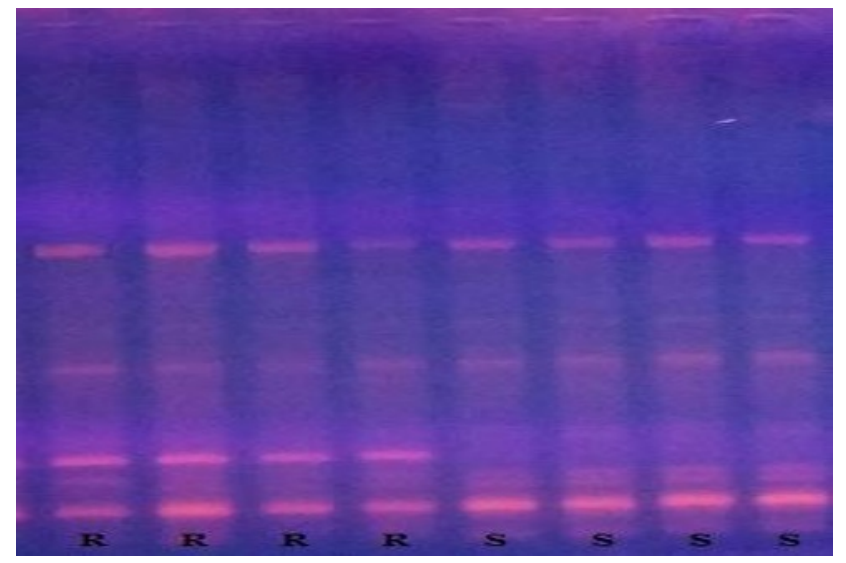

$\mathrm{R}$ (Resistant) - resistant isogenic line 269-Yas12p-2;

S (Susceptible)- susceptible isogenic line Pi714.

Fig. 1. Visualization of PCR products at the BoESSR 726 locus in 2\% agarose gel.

Figure 1 shows the allelic difference between contrasting genotypes (isogenic lines). Therefore, this marker will be used in our further studies on a segregating population to study its co-inheritance with this trait.

When studying the remaining 27 markers used in the study, no polymorphism was found. The results of testing 2 such markers are shown in Figure 2. From the presented electrophoregram, it can be seen that the bends in resistant and susceptible white cabbage samples are identical, therefore, these markers are not suitable for further breeding work on the basis of resistance to Xanthomonas campestris.

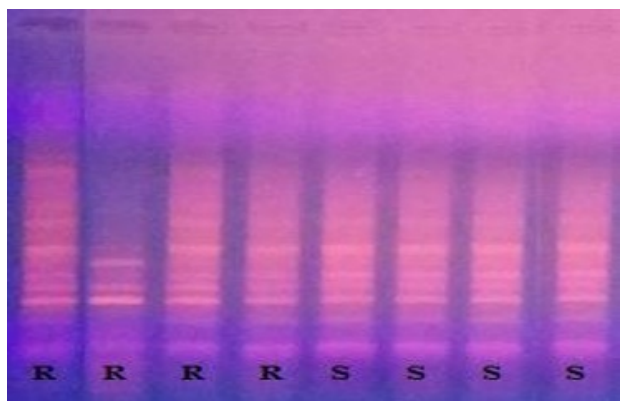

A

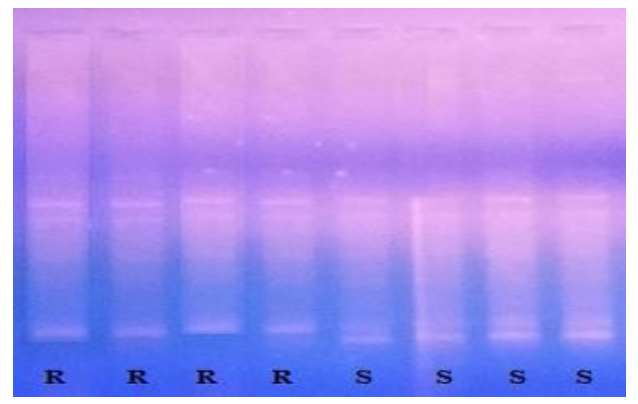

B

$\mathrm{R}$ (Resistant) - resistant isogenic line 269-Yas12p-2;

S (Susceptible) - susceptible isogenic line Pi714.

Fig. 2. A- Visualization of PCR products at the BoGMS 301 locus in $2 \%$ agarose gel; BVisualization of PCR products at the BoESSR 216 locus in $2 \%$ agarose gel.

At the next stage, the microsatellite markers Ol10-C01 and O111-H06, which revealed polymorphism according to the results of our earlier study [15], were tested on breeding samples of white cabbage (F2 plants of the hybrid combination 269-Yas12p-2 x Pi714) to check their co-inheritance with the resistance to Xanthomonas campestris, which is 


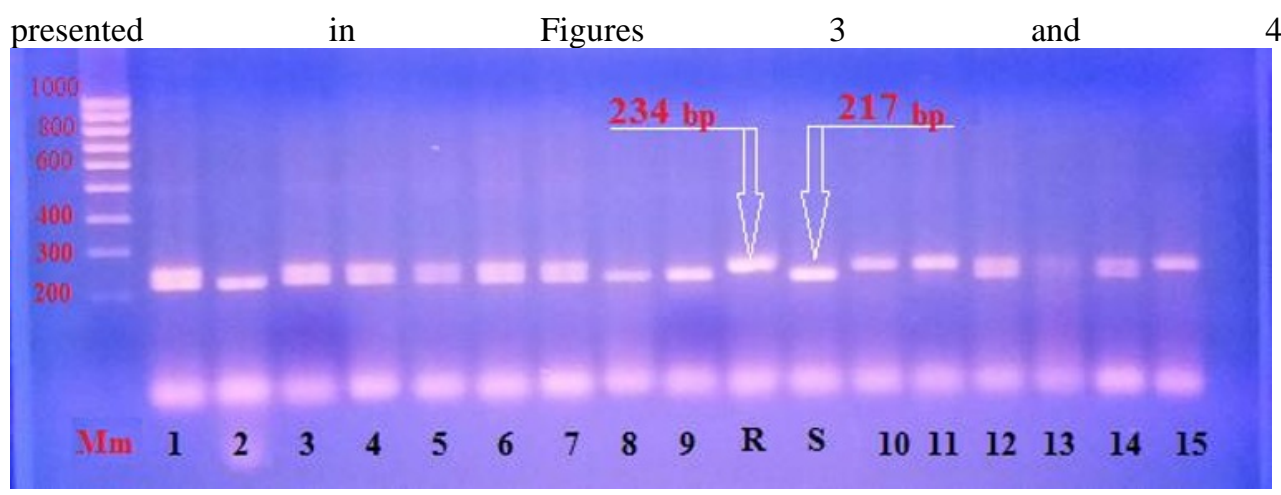

$\mathrm{Mm}$ - molecular mass marker; 1-15 - F2 plants of hybrid combination 269-Yas12p-2 x Pi714; $\mathrm{R}$ (Resistant)- resistant isogenic line 269-Yas12p-2;

S (Susceptible)- susceptible isogenic line Pi714.

Fig. 3. Separation of the F2 plant amplification products of the hybrid combination 269- Yas $12 p-2 x$ $\mathrm{Pi} 714$ at the Ol10-C01 locus.

Based on the electrophoretogram in Figure 3, it can be seen that already among the first analyzed 15 F2 plants, splitting is observed, and all types of genotypes are identified, i.e. plants $10,11,15$ are homozygous for the recessive allele (carry the allele of resistance), plants 2, 8, 9 are homozygous for the dominant allele of susceptibility, plants 1, 3-7, 12, 14 are heterozygous. The size of the alleles was also determined: the dominant susceptibility allele has a PCR product of $217 \mathrm{bp}$, and the donor resistance allele has $234 \mathrm{bp}$.

Currently, DNA analysis of the rest of the F2 plants at this locus is being carried out to obtain the ratio of homo- and heterozygotes and check its correspondence to the Mendelian 1: $2: 1$.

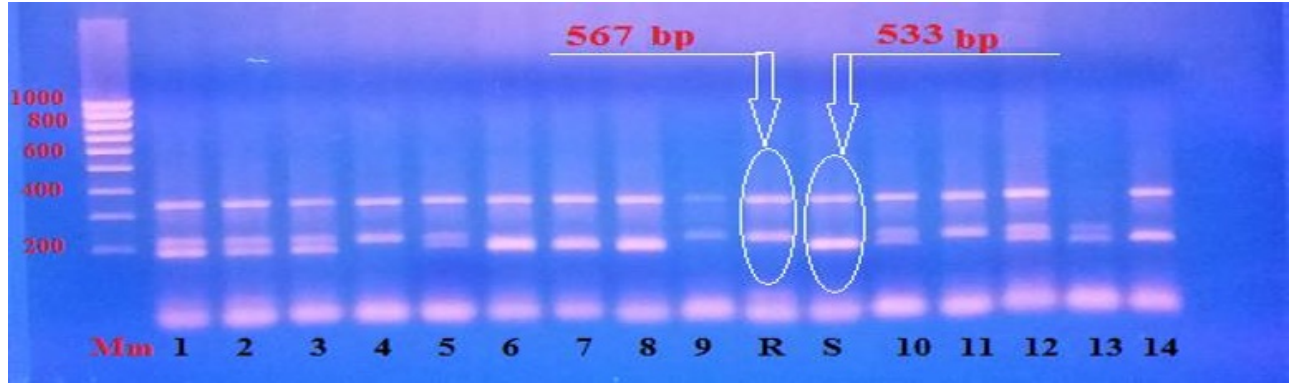

Mm - molecular mass marker; 1-14 - F2 plants of hybrid combination 269-Yas12p-2 x Pi714;

$\mathrm{R}$ (Resistant) - resistant isogenic line 269-Yas12p-2;

S (Susceptible) - susceptible isogenic line Pi714.

Fig. 4. Separation of plant amplification products of the isogenic line 269-Yas12p-2 at the O111-H06 locus.

Figure 4 shows the allelic diversity between the studied F2 plants. Here, just as at the Ol11-H06 locus, plant splitting by genotype is observed, i.e. plants numbered 4, 9, 11 are homozygotes for the recessive allele (resistance donors), plants: 6, 7, 8, 14 are homozygotes for the dominant allele (susceptible samples), and plants: 1, 2, 3, 5, 10, 12, 13 are heterozygous. It was found that the donor resistance allele has a PCR product of $567 \mathrm{bp}$, the susceptibility allele has $533 \mathrm{bp}$.

In the future, all remaining F2 plants will also be analyzed using this marker, phytopathological testing of these samples will be carried out, and subsequently the final 
ratio by genotype and phenotype will be drawn up, on the basis of which it will be possible

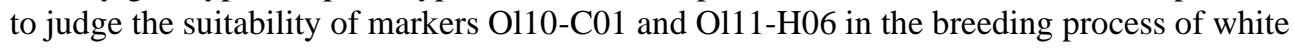
cabbage for resistance to black rot.

\section{Conclusions}

Based on the results of the studies, 3 SSR markers (O110-C01, O111-H06, BoESSR 726) were selected, showing polymorphism in the forms of white cabbage contrasting in resistance to black rot. Also markers O110-C01, Ol11-H06 reveal all types of allelic state in F2 plants. Therefore, the work with these markers will be continued on the segregating population to study their co-inheritance with the trait of resistance to black rot.

\section{References}

1. D. Singh, B.T. Raghavendra, P. Singh Rathaur, H. Singh, R. Raghuwanshi, R.P. Singh, Seed Sci. \& Technol., 42, 36-46, (2014) http://doi.org/10.15258/sst.2014.42.1.04

2. N.N. Tonu, M.A. Doullah, M. Shimizu, M.M. Karim, T. Kawanabe, R. Fujimoto, K. Okazaki, American Journal of Plant Sciences 4, 11-20 (2013) http://dx.doi.org/10.4236/ajps.2013.48A002

3. Y. Kifuji, H. Hanzaea, Y. Terasawa, T. Nishio, Euphytica 190, 289-295 (2013) https://doi.org/10.1007/s10681-012-0847-1

4. J.G.Vicente, E.B. Holub, Mol. Plant Pathol. 14(1), 2-18 (2013) https://doi.org/10.1111/j.1364-3703.2012.00833.x.

5. Vo Thi Ngok Ha, F.S. Dzhalilov, E.S. Mazurin, E.I. Kyrova, S.V. Vinogradova, A.N. Ignatov, Zasch. Kart. 2, 26-28 (2014) http://www.kartofel.org/zakart/zakart2_2014.pdf

6. S.V. Koroleva, S.A. Dyakunchak, S.A. Yurchenko, Veg. Crops Rus. 4, 16-20 (2019) https://doi.org/10.18619/2072-9146-2019-4-16-20

7. J. Cruz, R.Tenreiro, L. Cruz J., Plant Pathol. 9, 403-414 (2017) https://doi.org/10.4454/jpp.v99i2.3890.

8. B.B. Sharma, P. Kalia, D.K. Yadava, D. Singh, T.R. Sharma, PLoS ONE 11(3) (2016) https://doi.org/10.1371/journal.pone.0152290

9. K.S. Afrin, M.A. Rahim, J. Park , S. Natarajan, M.H. Rubel, H. Kim, I. Nou, Plant Breed. Biotech. 6(1), 30-43 (2018) https://doi.org/10.9787/PBB.2018.6.1.30

10. J. Lee, N.K. Izzah, V. Jayakodi, S. Perumal, H.J. Joh, H.J. Lee, S. Lee, J.Y. Park, K. Yang, I. Nou, J. Seo, J. Yoo, Y. Suh, K. Ahn, J.H. Lee, G.J. Choi, Y. Yu, H. Kim, T. Yang, BMC Plant Biol. 15(32) (2015) https://doi.org/10.1186/s12870-015-0424-6.

11. K.S. Afrin, M.A. Rahim, J. Park, S. Natarajan, H. Kim, I. Nou, Mol. Biol. Rep. 45(5), 773-785 (2018b) https://doi.org/10.1007/s11033-018-4217-5.

12. E.V. Dubina, V.N. Shilovsky, P.I. Kostylev, M.G. Ruban, A.M. Ogly, Plant Biotech. and Breed.2(1) (2019) https://doi.org/10.30901/2658-6266-2019-1-16-23

13.E.V. Dubina, A.V. Alabushev, P.I. Kostylev, Yu.A. Makukha, M.G. Ruban, I.V. Balyasnyi, Le.H. Ham, D.X. Tu, Le.H. Linh, African Journ. of Agricult. Research 13(48), 2757-2762 (2018) https://doi.org/10.5897/AJAR2018.13563.

14. J.F. Li, L. Li, J. Sheen, Plant Methods 6(1) (2010) http://dx.doi.org/10.1186/1746-48116-1

Makukha,

BIO Web

Conf.

21

(2020)

https://doi.org/10.1051/bioconf/20202100013 